\section{p38 kinase inhibitor approved for idiopathic pulmonary fibrosis}

The first treatment for the debilitating and fatal lung disease idiopathic pulmonary fibrosis (IPF) has been approved in Europe. On March 3, the European Commission gave InterMune of Brisbane, California, the go-ahead to market the orphan drug Esbriet (pirfenidone) - a rare success in a disease that has recently thwarted drug development efforts at several companies. In December, Gilead Sciences of Foster City, California, suspended phase 3 studies in IPF for Letairis (ambrisentan), which was already approved to treat pulmonary arterial hypertension. Nine months earlier, Swiss biotech Actelion suspended a similar compound Tracleer (bosentan; also previously registered for pulmonary arterial hypertension) when it failed to show significance in phase 3 studies of IPF. Whereas the new drug Esbriet targets the p38 gamma kinase, Tracleer and Letairis both target the endothelin system.

Getting the nod for Esbriet has not been without hitches - at least in Western markets. The US Food and Drug Administration refused to approve the small molecule last May, issuing InterMune with a complete response letter asking for an additional clinical trial. In 2008, under the trade name Pirespa, Osaka, Japan-based Shionogi had previously obtained approval for the use of pirfenidone against IPF in Japan. According to InterMune CEO Daniel Welch, the European approval has been built on the company's experience in IPF. Over the past 11 years, he says "we've studied more patients than any other company."

Welch says one of the key difficulties in developing treatments has been in understanding the definition of the disease, in which the generation of fibrotic tissue composed of extracellular matrix (ECM) proteins, including collagen and fibronectin, incrementally - and fatally - reduces the oxygen-exchanging capacity of the lungs. "The medical community doesn't understand what causes IPF. If it did, it would be a lot easier trying to figure out how to stop it," Welch says.

InterMune and others have kept trying (Table 1), not only because of the unmet medical need-IPF accounts for $\sim 135,000$ patients in Europe and 200,000 in the US-but also because an effective treatment for this orphan disease potentially would open the door to treatments of many other fibrotic diseases.

Esbriet is a small-molecule $\mathrm{p} 38$ gamma kinase inhibitor that blocks the synthesis

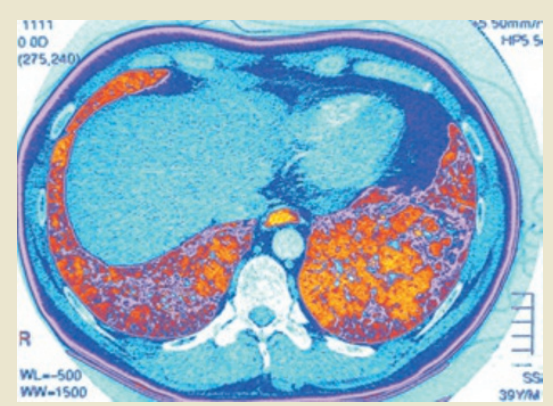

Scan showing pulmonary fibrosis with scarred

of transforming growth factor beta (TGF- $\beta$ ), which is involved in the process of producing fibrotic tissue. Stromedix, located in Cambridge, Massachusetts, also has TGF- $\beta$ in its sights. The company expects to launch a phase 2 trial of STX-100, a humanized monoclonal antibody ( $\mathrm{mAb}$ ) against integrin $\alpha-v-\beta-6$, an ECM protein that controls the activation of TGF- $\beta$, later this year.

Another human $\mathrm{mAb}$ for the treatment of IPF currently in phase 2 is FG-3019, which was developed using Medarex's UltiMAb system (Nat. Biotechnol. 27, 781-783, 2009). This connective tissue growth factor (CTGF) blocker is being developed by FibroGen of San Francisco. The approach is based on the observation that CTGF promotes myofibroblast formation, which in turn stimulates excessive production of the ECM proteins in fibrotic tissue. FibroGen's CEO Thomas Neff, notes that CTGF is at the center of multiple feedback loops that drive the fibrosis process, regardless of how it is initiated. "Other approaches target modification of indirect fibrogenic factors, or a single point in the process," he says.

Promedior of Malvern, Pennsylvania, claims its recombinant human protein Pentraxin-2 (PTX-2) targets a wide range of pro-fibrotic pathways driven by TGF- $\beta$. lung tissue.
"Fibrosis is a very complex pathology [prompted by] multiple redundant stimuli," says Promedior CEO Nick Colangelo. "If you just target one, you are not likely to be effective." Preclinical data published in February 2011 show PTX-2, also known as human serum amyloid $P$, inhibits airway inflammation, apoptosis, pulmonary fibrocyte and M2 macrophage accumulation, and collagen deposition.

The endothelin system-the focus of drug development programs at Gilead and Actelion-is thought to be a promising drug target in IPF because endothelin signals the synthesis and deposition of ECM components and contractile ability involved in lung fibrosis. Despite its recent setback with Letairis, a small-molecule antagonist of the endothelin A receptor, Gilead maintains its interest in IPF. The biotech gained access to AB0024, a humanized $\mathrm{mAb}$ that allosterically inhibits the enzyme lysyl oxidase-like 2 (LOXL2), through the acquisition of Arresto Biosciences last December. LOXL2 cross-links collagen to generate the scaffold on which fibroblasts grow, and in preclinical studies AB0024 has been shown to reduce levels of activated fibroblasts.

Despite its previous failure in IPF with Tracleer, a small-molecule inhibitor of both endothelin A and B receptors, Swiss biotech Actelion is also continuing development efforts in the disease. In collaboration with Nippon Shinyaku of Kyoto, Japan, the company is now taking through the clinic macitentan, another small-molecule antagonist of the dual endothelin receptors. Phase $2 b$ resoults for macitentan are due later this year. The company says this dual endothelin receptor inhibitor is more potent and has lower liver toxicity than Tracleer. Nuala Moran London

Table 1 Selected therapies for idiopathic pulmonary fibrosis in development

\begin{tabular}{|c|c|c|c|}
\hline Company & Compound & Mechanism & Status \\
\hline InterMune & Esbriet (pirfenidone) & Small-molecule p38 gamma kinase inhibitor & $\begin{array}{l}\text { Approved } \\
\text { in Europe }\end{array}$ \\
\hline Johnson \& Johnson & CNTO 888 & mAb against human $\mathrm{CC}$ chemokine ligand 2 & Phase 2 \\
\hline FibroGen & FG-3019 & $\begin{array}{l}\text { Fully human mAb blocking connective tissue } \\
\text { growth factor }\end{array}$ & Phase 2 \\
\hline Actelion & Macitentan & $\begin{array}{l}\text { Endothelin receptor type A, Endothelin } \\
\text { receptor type B }\end{array}$ & Phase 2 \\
\hline Novartis & QAX-576 & Interleukin-13 Receptor (IL-13R) & Phase 2 \\
\hline Stromedix & STX-100 & $\begin{array}{l}\text { Humanized } \mathrm{mAb} \text { against integrin alpha-v } \\
\text { family }\end{array}$ & Phase $1 / 2$ \\
\hline Gilead & GS-6624 (AB0024) & $\begin{array}{l}\text { Humanized mAb targeting human lysyl } \\
\text { oxidase-like- } 2 \text { protein }\end{array}$ & Phase 1 \\
\hline Promedior & Pentraxin-2 (PTX-2) & Recombinant human Pentraxin & Phase 1 \\
\hline
\end{tabular}

